\title{
Reducing the psychological distress of family caregivers of home based palliative care patients: longer term effects from a randomised controlled trial
}

\author{
Peter Hudson 1,2,3*, Tom Trauer ${ }^{4,3,5}$, Brian Kelly ${ }^{6}$, Moira O'Connor ${ }^{7,8}$, Kristina Thomas', Rachel Zordan' \\ and Michael Summers ${ }^{9}$ \\ 'Centre for Palliative Care, St. Vincent's Hospital, Melbourne, Australia \\ ${ }^{2}$ Queen's University, Belfast, Northern Ireland, UK \\ ${ }^{3}$ University of Melbourne, Melbourne, Victoria, Australia \\ ${ }^{4}$ Psychiatry, St. Vincent's Hospital, Melbourne, Victoria, Australia \\ ${ }^{5}$ Monash University, Melbourne, Victoria, Australia \\ ${ }^{6}$ Psychiatry, University of Newcastle, Callaghan, New South Wales, Australia \\ ${ }^{7}$ WA Centre for Cancer and Palliative Care and Curtin Health Innovation Research Institute, Curtin University, Perth, Western Australia, Australia \\ ${ }^{8}$ School of Psychology and Social Science, Edith Cowan University, Joondalup, Western Australia, Australia \\ ${ }^{9}$ Centre for Health Communication and Participation, La Trobe University, Melbourne, Victoria, Australia
}

* Correspondence to:

Centre for Palliative Care, St. Vincent's Hospital, Melbourne, Australia.

E-mail: phudson@unimelb.edu.au

Received: 10 October 2013

Revised: 5 May 2014

Accepted: 3 June 2014

\begin{abstract}
Background: Palliative care incorporates comprehensive support of family caregivers because many of them experience burden and distress. However, evidence-based support initiatives are few.

Purpose: We evaluated a one-to-one psychoeducational intervention aimed at mitigating the distress of caregivers of patients with advanced cancer receiving home-based palliative care. We hypothesised that caregivers would report decreased distress as assessed by the General Health Questionnaire (GHQ).

Method: A randomised controlled trial comparing two versions of the delivery of the intervention (one face-to-face home visit plus telephone calls versus two visits) plus standard care to a control group (standard care only) across four sites in Australia.

Results: Recruitment to the one visit condition was 57, the two visit condition 93, and the control 148. We previously reported non-significant changes in distress between times 1 (baseline) and 2 (1-week post-intervention) but significant gains in competence and preparedness. We report here changes in distress between times 1 and 3 (8-week post-death). There was significantly less worsening in distress between times 1 and 3 in the one visit intervention group than in the control group; however, no significant difference was found between the two visit intervention and the control group.

Conclusions: These results are consistent with the aim of the intervention, and they support existing evidence demonstrating that relatively short psychoeducational interventions can help family caregivers who are supporting a dying relative. The sustained benefit during the bereavement period may also have positive resource implications, which should be the subject of future inquiry.

(C) 2014 The Authors. Psycho-Oncology published by John Wiley \& Sons Ltd.
\end{abstract}

\section{Introduction}

Support for family caregivers is a core aspect of palliative care provision [1]. Family caregivers provide the bulk of support to patients receiving palliative care; without this assistance, patients' well-being and capacity to remain at home would be compromised [2]. Family caregivers are prone to negative psychological, physical, social and financial sequelae [3]. Depression rates of between 12 and 59\% [4-6] and anxiety rates of between 30 and 50\% $[5,6]$ have been reported. Although family caregivers are commonly referred to as 'hidden patients' [7], the support that they receive from health services is often unsystematic and incomplete [8]. Resource issues in rural areas add to the challenges of providing optimal family-centred support [9].

Systematic reviews of family caregiver interventions reveal that effective supportive care strategies are underdeveloped [10-12]. Effect sizes tend to be small to very small [11]. A recent international study [13] recommended that interventions should focus on carers' unmet needs, and one of the most consistently reported unmet needs relates to psychological support [14]. In addition to various forms of information, family caregivers want strategies to minimise their own psychological burden [15]. 
The current study built on a theoretical framework and pilot research showing family caregivers of patients with advanced cancer who received a home-based psychoeducational intervention (aimed at preparing them for the role of supporting a dying relative) reported a significantly more positive experience both while caregiving and 8 weeks after their relative's death [16]. However, it concluded that future studies should include larger samples and focus more on strategies to reduce psychological distress.

In keeping with this background, we tested (via a phase III randomised controlled trial) the following hypothesis: Primary family caregivers of patients with advanced cancer receiving palliative care at home (standard care) who also participate in a psychoeducational intervention will report decreased levels of psychological distress. We recently showed [17] that the intervention had small but non-significant effects on distress but significant improvements in participants' levels of preparedness and competence in one form of the intervention, between referral to the palliative service and 1 week after the intervention. We now report the changes in distress between referral to palliative care and 8 weeks after the patient's death.

\section{Methods}

The methods have been described in detail previously [17] and are presented here in abbreviated form. Time one (baseline) was within 2 weeks of referral to palliative care, time two was 1 -week post-intervention (5-week postrecruitment), and time three was 8 weeks after the patient's death. Time 1 data were collected in the family caregiver's home after obtaining informed consent and times 2 and 3 via mail-out self-report questionnaires with a follow-up reminder telephone call as required.

The sample consisted of adult, English-speaking primary family caregivers of patients with advanced cancer receiving home-based palliative care. Caregivers of patients with a non-malignant diagnosis or a poor functional status (using a standardised measure [18]) indicating likelihood of imminent death (as assessed by a treating clinician) were excluded in order to reduce attrition. We recruited from four home-based palliative care services in three states of Australia (Victoria, New South Wales, and Western Australia) over a 2-year period (2009-2011).

Usual care in these settings was explored prior to commencement [8] and comprised multidisciplinary specialist support for patients with advanced, non-curative disease and their families. Services included an initial assessment, scheduled home visits and access to a health care professional after hours for advice. Specific caregiver support strategies varied within services and were not always systematic or comprehensive.

Those randomised to one of the two experimental groups received standard care, plus a psychoeducational intervention that incorporated tailored information and resources given to family caregivers to promote psychological well-being. The intervention was delivered in two versions given that in rural and remote areas where, because of limited resources, telephone contact rather than face to face may be more feasible. The difference between the two versions was in the form of contact only. Intervention 1 consisted of one visit and three phone calls, and intervention two consisted of two visits and two phone calls. For both groups, the first contact was a face-to-face visit, and in the latter group, the final contact was also a home visit.

Each caregiver was allocated a Family Caregiver Support Nurse (FCSN) who assisted the local palliative care service to assess caregiver needs, establish a care plan and provide additional caregiver support. The FCSNs (one per recruitment site) received training from relevant members of the research team, and an intervention manual was developed to foster consistency in delivery. The primary written resource for caregivers was a guidebook, developed and tested in our pilot work [19] that focused on preparation and management of the caregiver role with a strong focus on promoting psychological well-being. Both versions of the intervention were delivered over 4 weeks and comprised the following: (a) preparing caregivers for participation in the intervention, (b) assessing caregiver needs and preparing a care plan, (c) re-assessing needs and evaluating the care plan, and (d) assisting the family caregiver to prepare for their relative's death and to prepare for bereavement. The FCSN concluded the structured component of the intervention (both versions) with a summary (including a written format) of key strategies and resources. The care plan was revised, incorporating referral to other services as required.

Participants were randomised to either the control or intervention groups. For rural sites, the allocation ratio was set at 3:2:1 (control : two visits: one visit). Having regard for some one visit interventions needing to be conducted in metropolitan sites, the allocation ratio was set at 3:1:2 (control: one visit: two visits). The research assistants responsible for data collection were blinded to group allocation in order to minimise response bias.

The primary outcome variable was psychological distress measured by the General Health Questionnaire (GHQ) - 12 items [20] at all three time points. Scores for items were totalled, based on ordinal responses (0-3) with higher scores indicative of greater levels of distress. The GHQ has established reliability and validity [20]. We used the ordered category scoring system, which can assess the intensity of symptoms as well as psychiatric caseness [21], an approach that Andrich and van Schoubroek [22] showed to be satisfactory. Secondary outcomes, not assessed at the final data collection, included measures of caregiver competence, preparedness, needs, and rewards, as detailed in our earlier publication [17]. Participants also provided sociodemographic information. 
Full details of sample size considerations are presented in Hudson and colleagues' initial publication linked to this study [17]. Conventional levels of statistical significance (alpha $=.05)$ and minimum power $(0.80)$ were adopted. Anticipating an effect size of at least .5 yielded a requirement of 168 participants in total. After allowing for expected attrition, a sample size of 240 was estimated to be sufficient.

Primary analyses were undertaken on an intention to treat (ITT) basis, including all participants as randomised, regardless of completion or extent of participation in the intervention. Overall effectiveness of the intervention was evaluated using a planned contrast within a mixedmodel repeated measure (MMRM) analysis [23]. The contrast compared change from time 1 to time 3 under the active intervention, regardless of the mode of delivery to change in the placebo arm. A subsidiary comparison compared change between modes of delivery. Withinparticipant covariation was modelled by an unconstrained variance-covariance matrix. MMRM was preferred over conventional methods of analysis because it retains information from all participants, including those with missing observations at one or more points, yielding an ITT estimate under mild assumptions [24]. Effect sizes were computed using the estimated means and the time 1 control group standard deviation as the denominator.

The time $1 \mathrm{GHQ}$ scores were found to have a significant departure from a normal distribution $\left(\chi_{(2)}^{2}=15.42, p<.001\right)$ by a joint test of skew and kurtosis [25]. Following a square root transformation, the joint test was no longer statistically significant $\left(\chi^{2}{ }_{(2)}=2.94, p=.23\right)$. On this basis, we used the square root of GHQ (sGHQ) in analyses.

\section{Results}

\section{Recruitment and demographics}

Figure 1 includes information relating to the following: numbers approached, eligibility, recruitment, allocation to each study condition and data collected for the three time points. Of the 422 who were ineligible, reasons included the following: patient imminently dying (79), planned to consent but patient had died (133), non-English speaking (53), impossible to contact (140), or 'other' reasons (17). Reasons for eligible people declining participation (441) are the following: too busy (190), not interested (59), too overwhelmed (67), 'other' reasons (105) and no reason (20).

Of the 741 who were eligible, 298 participants were recruited (40\%). Of the 298, 49 were recruited from rural sites and 249 from metropolitan sites. Two hundred and seventy-seven were assessed at time 1 and 160 at time 3, a loss of $42 \%$. As for loss/gain, after randomisation, a few participants were lost, and thereafter, participants could be lost or regained at any time point. Reasons for

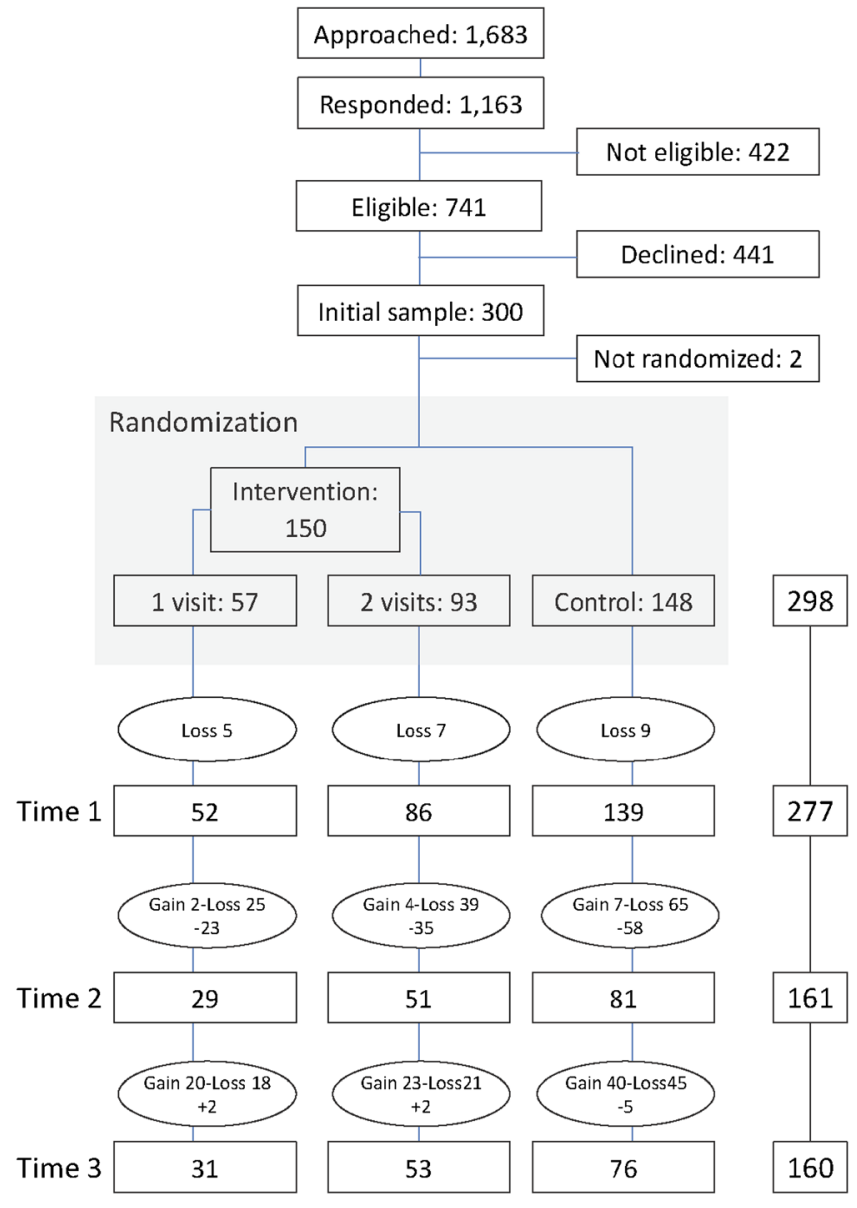

Figure I. Consolidated Standards of Reporting Trials diagram

attrition included the following: patient no longer met the inclusion criteria $(n=22)$, patient died before time 2 $(n=9)$, or the carer withdrew from the study $(n=17)$. However, in the majority of circumstances $(n=80)$, the reason(s) were not identified. Difference in age, gender, mean scores on the GHQ and other measures at time 1 between those who had time 3 data and those who did not was all non-significant.

Two hundred and eleven participants were female patients and 85 male patients, and for 2 participants, gender was not recorded. The percentage of women (about 70\%) was similar in both study arms (control and intervention), but there were more women (75\%) in intervention 2 than in intervention $1(60 \%)$. This difference is nearly statistically significant $(p=.07)$. The mean age for the 264 for whom age was provided was 59.0 years $(\mathrm{SD}=13.9$, $\mathrm{min}$ 22 , max 88). The mean age of men (61.7) was significantly higher than women $\left(57.9 ; t_{(262)}=2.02, p=.044\right.$ two-tailed $)$.

The majority of participants were caring for a spouse/ partner $(54 \%)$ followed by a parent (24\%), child (13\%), other relative $(16 \%)$, or friend (14\%). Over three quarters (79\%) lived with the person that they were caring for and, on average, had been caring for this person for 6.2 years $(\mathrm{SD}=9.43$ years, range $1-60$ years). Many participants 
had reduced work $(21 \%)$ or stopped work $(24 \%)$ in order to provide care; however, $16 \%$ were still employed in some capacity. None of the demographic variables varied significantly via intervention group.

The median interval between the collection of time 1 and time 2 data was 42 days, and the median interval between the collection of time 2 data and the patient's death was 45 days. Time 3 questionnaires were sent out approximately 8 weeks after the patient's death; however, because of the need for reminders, the median time to receiving time 3 data was 96 days. At time 1, there were 275 GHQ assessments; the mean raw GHQ score was 14.8 (SD 5.6); the means of the control, one visit, and two visit groups were $15.0,14.7$ and 14.5 respectively. Using the threshold for psychiatric caseness of 13/14 determined by Piccinelli et al. [26], the proportions of possible cases among participants in the three study conditions at time 1 were between 51 and $55 \%$.

\section{Intervention effects}

Age and gender were included in the analysis for several reasons. While the mean ages of participants in the three study conditions were not significantly different, research has found that older people tend to score higher (worse) than younger [27], while in the present data, the reverse was true (data not shown). Gender was nearly significantly different between the three study conditions and was also found by McCabe et al. [27] to be related to GHQ score. Figure 2 shows the estimated means of the three study conditions at the three time points.

The results showed significant effects for age $(p<.001)$ and gender $(p=.04)$, a near significant effect of time $(p=.06)$, and the overall interaction of intervention with time was not significant $(p \geq .15)$. The age and gender effects indicate that, on average, younger participants, and

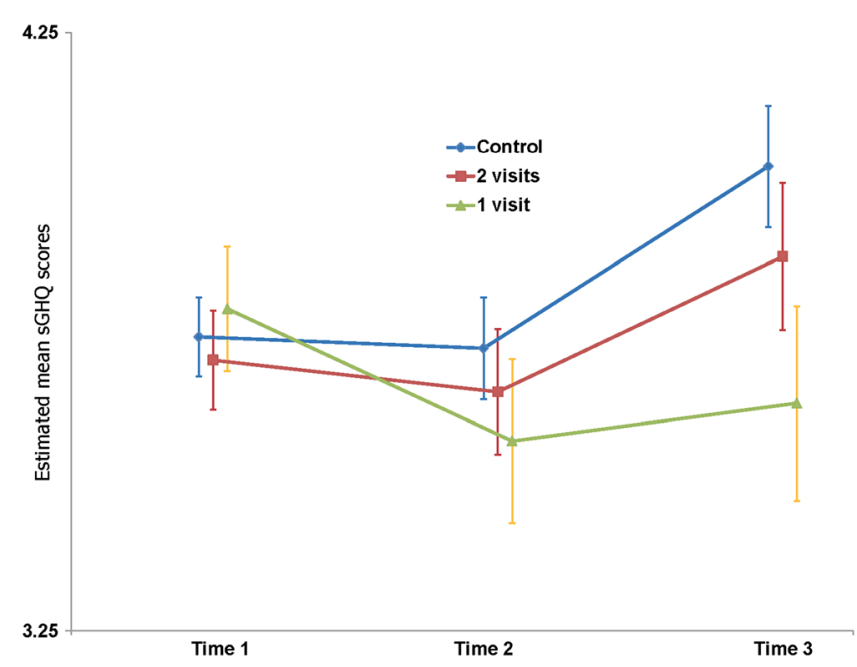

Figure 2. Estimated mean sGHQ scores, with standard error bars, at times I, 2 and 3, by study condition females, had higher (worse) scores. The planned contrast of the difference in change between times 1 and 3 in the intervention groups versus the control group was significant $\left(t_{(150)}=2.03, p=.044\right)$, while the contrast of the difference in change over time between the two modes of delivery was not significant $\left(t_{(149)}=1.72, p=.09\right)$. Despite the latter outcome, Figure 2 clearly shows a divergence between the two forms of the intervention not apparent at time 1. Contrast change between the control group, and (a) the two visit group and (b) the one visit group was not significant $(t$ (149) $=0.74, p=.45$, and $t_{(150)}=2.42, p<.02$ respectively). Considered as post hoc comparisons, the latter remains significant $(p<.05)$ after Bonferroni adjustment. The effect sizes for the one visit group, the two visit group, and the two groups combined relative to the control group were $.58, .15$, and .36 respectively. Using Cohen's [28] guidelines for effect sizes ( .2 small, .5 medium, and $\geq .8$ large), we see that there are small to medium effects of the interventions relative to the control group. We conclude that, relative to participants in the control group, the sGHQ scores of participants in the intervention conditions worsened less. This is supported by a comparison of the changes between time 1 and time 3 within each of the experimental conditions. The estimated mean in the control group rose significantly $\left(0.28 ; t_{(150)}=2.97, p=.003\right)$. However, the mean of the one visit group fell non-significantly $\left(-0.15, t_{(150)}=1.02\right.$, $p=.33$ ), while that of the two visit group rose non-significantly $\left(.17 ; t_{(149)}=1.51, p=.13\right)$. These results indicate that the intervention mitigates the commonly experienced rise in distress after the patient has died but, not surprisingly, does not fully prevent distress.

\section{Discussion and conclusions}

We previously showed [17] that general distress, as assessed by the GHQ, worsened somewhat less, and non-significantly, in the intervention groups relative to the control group in the interval between baseline and 1 week after the end of the intervention. We have now shown that by 8 weeks after the patients' deaths, distress among carers in the control group increased more than among carers in the intervention groups; significantly so under the one visit delivery mode. Thus, our main hypothesis was supported. Overall, the intervention had a significant small to medium-sized effect. However, the significant effect of the combined group was largely because of the effect of the one visit condition. Carers in both conditions were contacted the same number of times; however, those allocated to the one visit condition received one face-to-face contact and three telephone calls, whereas those allocated to the two visit condition received two face-to-face contacts and two telephone calls. Our result is not inconsistent with the preponderance of the literature demonstrating that face to face and telephone contact are equivalent in terms of assessment 
of mental states [29,30] and behavioural interventions [31,32]. Demiris and colleagues found that a problemsolving therapy for hospice caregivers delivered via video was not inferior to face-to-face delivery [33]. There is, however, a suggestion of superiority of the one visit condition over the two visit mode. This needs replication, but we can speculate as to what could account for the difference. One reason that the final delivery of the final phase of the intervention by phone may have been more helpful is that the carers may be more able to talk freely with the FCSN and voice their concerns and needs. It was noted that sometimes when the FCSN came to the house, the intervention often took place in the presence of the patient. The FCSN also reported difficulty getting the carers to appreciate that they were there to support the carer not the patient, which was lessened when the intervention took place over the phone and the focus was on the carer. It may also be that a phone contact is perceived as less intrusive; carers sometimes felt obliged to make preparations for the arrival of the nurse on the home visit.

Psychoeducational interventions may have negative effects, or the control group deteriorates whilst the intervention group remains stable [34]. An important outcome of the intervention studied here was the apparent absence of negative sequelae for caregivers in the intervention group.

Research implications and the limitations associated with this research are comprehensively outlined in our earlier publication [17]. However, with regard to specific matters arising from the longer term data, we make the following points. First, assessment of any enduring effects of interventions should be undertaken, for example, at 6-month postbereavement. Second, the potential impact of the intervention on reducing complicated/prolonged grief should be explored [35]. Third, based on the present findings, it would seem that the one visit intervention was more effective than the two visit version. This has favourable resource implications. Finally, it would be advantageous for future work to attempt to discern whether or not there are specific intervention ingredients that are more potent than others.
In conclusion, we have shown that a tailored psychoeducational intervention aimed at improving information and promoting well-being had some protective effect against the increase in distress associated with the death of a relative. In our earlier report [17], we concluded that this effect was not apparent immediately after the delivery of the intervention, but here, we have shown the effect to be present 8 weeks after the patient's death. It would be of interest to see whether the effects endured beyond this fairly short period. At this stage, explanations of apparent superiority of the one visit form of intervention over the two visit version must be regarded as speculative, given that their equivalent content, medium and circumstances of delivery appear to be critical, and this should be a focus for future research.

\section{Acknowledgements}

Sadly, Professor Tom Trauer (co-author and co-investigator on this research project), recently died unexpectedly. His input into this and other research was greatly appreciated.

Linda Kristjanson, Swinburne University, Victoria, Australia

Chris Hall, Australia Centre for Grief and Bereavement, Victoria, Australia

Kelli Stajduhar, University of Victoria, Canada

Anne Oakley, Melbourne Citymission Palliative Care, Victoria, Australia

Steve Carmody, Silver Care Hospice, Western Australia, Australia

Ruth Jones, Greater Western Area Health Service, New South Wales, Australia

Victoria White, the Cancer Council of Victoria, Australia

Helen Wearne, South East Palliative Care, Victoria, Australia

Andrew Mackinnon, University of Melbourne, Victoria, Australia

Research assistants and intervention nurses

Victorian Government Department of Human Services, Australia Supported by grant 447717 from the National Health and Medical Research Council (NHMRC), Commonwealth Department of Health and Aging, Australia. This project received approval from the relevant participating clinical sites.

The study was registered with the Australian and New Zealand Clinical Trials Registry (ANZCTR): trial ID ACTRN12608000621314, date registered 8 December 2008.

\section{References}

1. World Health Organisation. National cancer control programmes: policies and managerial guidelines. WHO: Geneva (Switzerland), 2002.

2. Stetz K, Brown M. Taking care: caregiving to persons with cancer and AIDS. Cancer Nurs 1997;20(1):12-22.

3. Rossi Ferrario S, Cardillo V, Vicario F, Balzarini E, Zotti AM. Advanced cancer at home: caregiving and bereavement. Palliat Med 2004;18(2):129-136.

4. Hauser JM, Kramer BJ. Family caregivers in palliative care. Clin Geriatr Med 2004;4:671-688.
5. Grunfeld E, Coyle D, Whelan TJ et al. Family caregiver burden: Results of a longitudinal study of breast cancer patients and their principal caregivers. Can Med Assoc J 2004; 170 (12):1795-1801.

6. Hudson P, Thomas K, Trauer T, Remedios C, Clarke D. Psychological and social profile of family caregivers on commencement of palliative care. J Pain Symptom Manage 2011;41 (3):522-534.

7. Kristjanson L, Aoun S. Palliative Care for Families: Remembering the hidden patients. Can J Psychiatry 2004;49(6):359-365.

8. Thomas K, Hudson P, Oldham L, Kelly B, Trauer T. Meeting the needs of family carers: an evaluation of three home-based palliative care services in Australia. $J$ Palliat Med 2010;24(2):183-191.

9. Van Vorst RF, Crane LA, Barton PL, Kutner JS, Kallail KJ, Westfall JM. Barriers to quality care for dying patients in rural communities. J Rural Health 2006;22(3):248-253.

10. Honea NJ, Britnall R, Given B et al. Putting Evidence into Practice: nursing assessment and interventions to reduce family caregiver strain and burden. Clin J Oncol Nurs 2008;12(3):507-516.

11. Candy B, Jones L, Drake R, Leurent B, King M. Interventions for supporting informal caregivers of patients in the terminal phase of a disease. Cochrane Database Syst Rev 2011;15(6):CD007617. PubMed PMID: 21678368. Epub 2011/06/17. eng. 
12. Hudson P, Remedios C, Thomas K. A systematic review of psychosocial interventions for family carers of palliative care patients. $B M C$ Palliative Care 2010;9(1):17. PubMed PMID: doi:10.1186/1472-684X-9-17.

13. Hudson P, Zordan R, Trauer T. Research priorities asssociated with family caregivers in palliative care: international perspectives. J Palliat Med 2011;14(4):397-401. PubMed PMID: 21375394. Epub 2011/03/08. Eng.

14. Harding R. Carers: Current research and developments. In: Facing Death: Loss, Change and Bereavement in Palliative Care. Facing Death, Firth P, Luff G, Oliviere D (eds.), 1st ed. Open University Press: Maidenhead, Berkshire, 2005; 150-166.

15. Kristjanson L, Hudson P, Oldham L. Working with families. In Palliative care nursing: A guide to practice, O’Connor M, Aranda S (eds.), 2 ed. Ausmed Publications: Melbourne, 2003; 271-283.

16. Hudson P, Aranda S, Hayman-White K. A psycho-educational intervention for family caregivers of patients receiving palliative care: A randomised controlled trial. J Pain Symptom Manage 2005;30(4):329-341.

17. Hudson P, Trauer T, Kelly B et al. Reducing the psychological distress of family caregivers of home based palliative care patients: Short term effects from a randomised controlled trial. Psycho-Oncology 2013;22(9):1987-1993.

18. Abernethy AP, Shelby-James T, Fazekas BS, Woods D, Currow DC. The Australiamodified Karnofsky Performance Status (AKPS) scale: A revised scale for contemporary palliative care clinical practice. $B M C$ Palliative Care 2005;4(7):1-12. DOI: $10.1186 /$ 1472-684X-4-7.

19. Hudson P. Supporting a person who requires palliative care: A guide for family and friends. Palliative Care Victoria: Melbourne (Australia), 2004.
20. Goldberg DP, Gater R, Sartorius N et al. The validity of two versions of the GHQ in the WHO study of mental illness in general health care. Psychol Med 1997;27(1):191-197. PubMed PMID: 9122299. Epub 1997/01/01. eng.

21. Goldberg D. The Detection of Psychiatric Illness by Questionnaire. Maudsley Monograph no. 21. Oxford University Press: London (UK), 1972

22. Andrich D, van Schoubroeck L. The General Health Questionnaire: a psychometric analysis using latent trait theory. Psychol Med 1989;19 (2):469-85. PubMed PMID: 2762448. Epub 1989/05/01. eng.

23. Brown H, Prescott R. Applied mixed models in medicine. (2nd ed.). Wiley: New York, 2006.

24. Hamer RM, Simpson PM. Last observation carried forward versus mixed models in the analysis of psychiatric clinical trials. Am J Psychiatry 2009;166(6):639-641.

25. StataCorp. Stata 11 Base Reference Manual. Release 11 ed: Stata Press, 2009.

26. Piccinelli M, Bisoffi G, Bon MG, Cunico L, Tansella M. Validity and test-retest reliability of the Italian version of the 12-item General Health Questionnaire in general practice: a comparison between three scoring methods. Compr Psychiatry 1993;34(3):198-205. PubMed PMID: 8339539. Epub 1993/05/01. eng.

27. McCabe CJ, Thomas KJ, Brazier JE, Coleman P. Measuring the mental health status of a population: a comparison of the GHQ-12 and the SF-36 (MHI-5). Br J Psychiatry 1996;169(4):516-21. PubMed PMID: 8894205. Epub 1996/10/01. eng.

28. Cohen J. Statistical power analysis for the behavioral sciences. 2nd ed. Lawrence Erlbaum: Hillsdale, 1988.
29. Kobak KA, Williams J, Jeglic E, Salvucci D, Sharp IR. Face-to-face versus remote administration of the Montgomery-Asberg Depression Rating Scale using videoconference and telephone. Depress Anxiety 2008;25(11):913-919.

30. Paing WW, Weller RA, Dixon TA, Weller EB. Face-to-face versus telephone administration of the parent's version of the children's interview for psychiatric syndromes (P-ChIPS). Curr Psychiatry Rep 2010;12(2): 122-6. PubMed PMID: 20425297.

31. Burgess M, Andiappan M, Chalder T. Cognitive behaviour therapy for chronic fatigue syndrome in adults: face to face versus telephone treatment: a randomized controlled trial. Behav Cogn Psychother 2012 Mar; 40(2):175-91. PubMed PMID: 21929831.

32. Opdenacker J, Boen F. Effectiveness of face-to-face versus telephone support in increasing physical activity and mental health among university employees. J Phys Act Health 2008;5(6):830-43. PubMed PMID: 19164818.

33. Demiris G, Parker Oliver D, Wittenberg-Lyles E et al. A Noninferiority Trial of a ProblemSolving Intervention for Hospice Caregivers: In Person versus Videophone. J Palliat Med 2012;15(6):653-60. PubMed PMID: 22536989. Pubmed Central PMCID: 3362957. Epub 2012/ 04/28. eng.

34. Acton GJ, Kang J. Interventions to reduce the burden of caregiving for an adult with dementia: a meta-analysis. Res Nurs Health 2001;24(5):349-360.

35. Guldin MB, Vedsted P, Zachariae R, Olesen $\mathrm{F}$, Jensen AB. Complicated grief and need for professional support in family caregivers of cancer patients in palliative care: a longitudinal cohort study. Support Care Cancer 2012;20(8):1679-1685. 


\section{University Library}

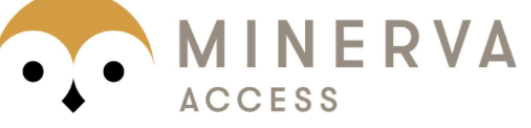

A gateway to Melbourne's research publications

Minerva Access is the Institutional Repository of The University of Melbourne

Author/s:

Hudson, P;Trauer, T;Kelly, B;O'Connor, M;Thomas, T;Summers, M;Zordan, R;White, V

Title:

Reducing the Psychological Distress of Family Caregivers of Home Based Palliative Care Patients: Short Term Effects From a Randomised Controlled Trial

Date:

2013-11-01

Citation:

Hudson, P., Trauer, T., Kelly, B., O'Connor, M., Thomas, T., Summers, M., Zordan, R. \& White, V. (2013). Reducing the Psychological Distress of Family Caregivers of Home Based Palliative Care Patients: Short Term Effects From a Randomised Controlled Trial. PSYCHOONCOLOGY, 22 (1), pp.76-76. https://doi.org/10.1002/pon.3610.

Persistent Link:

http://hdl.handle.net/11343/265687

License:

CC BY-NC-ND 